\title{
Philosophiques
}

\section{Claude Paris, Le discours religieux. Une réflexion, collection « Idées claires», Éditions C.G., Québec, 1988, 76 pages.}

\section{Jacques G. Ruelland}

Volume 17, numéro 2, automne 1990

URI : https://id.erudit.org/iderudit/027135ar

DOI : https://doi.org/10.7202/027135ar

Aller au sommaire du numéro

Éditeur(s)

Société de philosophie du Québec

ISSN

0316-2923 (imprimé)

1492-1391 (numérique)

Découvrir la revue

Citer ce compte rendu

Ruelland, J. G. (1990). Compte rendu de [Claude Paris, Le discours religieux. Une réflexion, collection "Idées claires», Éditions C.G., Québec, 1988, 76 pages.]

Philosophiques, 17(2), 228-229. https://doi.org/10.7202/027135ar d'utilisation que vous pouvez consulter en ligne.

https://apropos.erudit.org/fr/usagers/politique-dutilisation/ 
CLAUDE PARIS, Le discours religieux. Une réflexton, collection * Idées claires*, Éditions C.G., Québec, 1988, 76 pages.

par Jacques G. Ruelland

Ce manuel est divisé en trois chapitres intitulés * Une question de sens *, * Le discours religieux * et * La question de Dieu *. Chaque paragraphe du premier chapitre est une affirmation, sous laquelle l'étudiant(e) trouve cinq réponses possibles, dont il (ou elle) doit ensuite justifier le choix. Par exemple (p. 11):

Tous les miracles décrits dans la Bible sont vrais.

(Réponses :)

A. Le miracle constitue une irradiation (sic) éclairant notre monde selon des lois naturelles et transcendantes.

B. Je suis d'accord. La Bible ne peut pas contenir des comptes rendus inexacts de l'œuvre de Dieu.

C. Je ne suis pas d'accord; la compréhension des lois de la Nature (sic) et de la Société peuvent expliquer les * miracles *.

D. Je suis d'accord, mais uniquement au sens où le miracle désigne un récit voulant montrer la souveraineté de Dieu sur les processus naturels. Ils sont probablement inexacts dans les faits.

E. Je ne suis pas d'accord. Les miracles sont illusoires parce qu'il n'y a pas d'intervention divine dans la nature.

Intéressant, n'est-ce pas? L'athée aussi bien que le croyant y trouve son compte ! Et le professeur n'a plus qu'à animer le débat!

Le deuxième chapitre tente de définir l'essence du discours religieux sous les diverses formes de la croyance ou de l'attitude mystique. Le symbolisme du langage religieux est analysé dans ses aspects dogmatiques et rituéliques, ainsi que la pathologie de l'attitude religieuse: l'intégrisme, le fanatisme et la pensée magique. Le livre donne également des indications claires sur les diverses façons d'interpréter la Bible 
(littérale, littéraire, historique) et sur le «pouvoir * de la méditation comme voie d'expérience du sacré. Quelques exercices terminent cette partie de l'ouvrage.

Le chapitre le plus instructif est probablement le troisième: « La question de Dieu», où le sentiment religieux, l'agnosticisme et l'athéisme sont vus à la lumière de divers auteurs. Feuerbach, Marx et Nietzsche sont confrontés au théisme et au panthéisme. L'étudiant(e) est ainsi mis(e) dans l'obligation de se poser la question du fondement de sa propre croyance et d'y répondre par une argumentation bien étayée. L'examen des "preuves* de l'existence de Dieu, de la notion de miracle et du mythe de Sisyphe l'aident dans cette tâche ingrate. Des exercices et un choix de lectures terminent ce chapitre. Quelques textes d'auteurs (de toutes les tendances illustrées dans l'ouvrage) et un lexique complètent le manuel.

Ce livre est étonnant par sa recherche de neutralité. L'auteur prend soin de ne pas dévoiler ses propres convictions et de ne pas influencer celles de ses lecteurs et lectrices. Il reste à savoir si ce discours est aussi neutre qu'il le paraît.

Même si le professeur athée n'a pas toujours tendance à aborder la question de Dieu dans ses cours de philosophie, les étudiant(e)s I'y contraignent souvent... Il trouvera alors dans ce manuel un guide de discussion fort utile et très bien structuré, à l'aide duquel il pourra demander aux étudiant(e)s de remettre en question leurs convictions (souvent aveugles et indiscutées), et ce, dans le plus grand respect des un(e)s et des autres. Aucun autre livre ne lui sera nécessaire pour mener à bien ce débat.

\section{Collège Édouard-Montpetit}

